\title{
EFEITO DE PRÉ-CULTIVOS SOBRE O POTENCIAL DE INÓCULO DE FUNGOS MICORRÍZICOS ARBUSCULARES E PRODUÇÃO DA MANDIOCA ${ }^{1}$
}

\author{
FRANCISCO ADRIANO DE SOUZA², SANDRA FARTO BOTELHO TRUFEM ${ }^{3}$, DEJAIR LOPES DEALMEIDA ${ }^{4}$, \\ ELIANE MARIA RIBEIRO DA SILVA ${ }^{4}$ e JOSÉ GUILHERME MARINHO GUERRA ${ }^{5}$
}

\begin{abstract}
RESUMO - Avaliou-se o efeito de pré-cultivos com adubos verdes sobre o potencial de inóculo de fungos micorrízicos arbusculares e produção de mandioca (Manihot esculenta, Crantz). O experimento foi conduzido no campo experimental da Embrapa-Centro Nacional de Pesquisa de Agrobiologia (CNPAB), em solo Podzólico Vermelho-Amarelo, localizado no Município de Seropédica, RJ, em parcelas experimentais de $30 \mathrm{~m}^{2}$, dispostas em delineamento de blocos casualizados com quatro repetições. Os pré-cultivos constaram de Canavalia ensiformis, Mucuna aterrima, Crotalaria juncea, Cajanus cajan e Sorghum bicolor (BR 005) e o tratamento-controle (solo mantido sem plantas). Após um período de 82 dias de crescimento, sobre as mesmas parcelas cultivou-se mandioca, por oito meses. A produtividade de raízes de mandioca variou de 12,76 a 17,20 $\mathrm{t} \mathrm{ha}^{-1}$, porém não foi detectada diferença estatística entre os tratamentos. Foram identificadas 16 espécies de fungos micorrízicos arbusculares e foi constatado que o número de esporos de algumas espécies sofreu alteração da sua freqüência de ocorrência com os cultivos. O pré-cultivo com sorgo aumentou o número de propágulos infectivos em relação ao controle, demonstrando que o uso de espécies de plantas com capacidade para elevar o potencial de inóculo pode ser um bom caminho alternativo para resolver o problema de produção de inóculo para culturas micotróficas em campo.
\end{abstract}

Termos para indexação: Manihot esculenta, Canavalia ensiformis, Mucuna aterrima, Crotalaria juncea, Cajanus cajan, Sorghum bicolor, número de esporos, propágulos infectivos.

\section{EFFECT OF PRE-CROPS ON THE INOCULUM POTENTIAL OF ARBUSCULAR MYCORRHIZAL FUNGI AND CASSAVA YIELD}

\begin{abstract}
The effect of pre-crops on the inoculum potential (IP) of arbuscular mycorrhizal fungi (AMF) and yield of cassava was evaluated on a Red-Yellow Podzolic soil in the field. The experiment was carried out at Embrapa-Centro Nacional de Pesquisa de Agrobiologia (CNPAB), Seropédica, RJ, Brazil, in plots with $30 \mathrm{~m}^{2}$ arranged in a randomised block design with four replications. As pre-crops, four legume species were used: Canavalia ensiformis, Mucuna aterrina, Crotalaria juncea and Cajanus cajan; and a grass Sorghum bicolor (BR 005) in relation to plots, without plants. After a grow period of 82 days, at the same plots, cassava stakes were planted, which were grown for eight months. The yield of cassava roots ranged from 12.76 to 17.20 ton ha ${ }^{-1}$, although no statistical significant effects were found between the treatments. There were $16 \mathrm{AMF}$ species identified, and some of them had a significant frequency changement after the growing cycles. The number of infective propagules were significantly increased after sorghum crop in relation to plots without plants. It was showed that identification of plant species capable to increase the IP in the field soils may be a good alternative way to solve the problem of inoculum production to mycotrophic crops in the field.
\end{abstract}

Index terms: Manihot esculenta, Canavalia ensiformis, Mucuna aterrima, Crotalaria juncea, Cajanus cajan, Sorghum bicolor, infective propagule, number of spores.

\footnotetext{
${ }^{1}$ Aceito para publicação em 25 de fevereiro de 1999. Extraído da Tese de Mestrado do primeiro autor, apresentada à UFRRJ. Trabalho parcialmente apresentado na V Reunião Brasileira Sobre Micorrizas, Florianópolis, 1994.

${ }^{2}$ Eng. Agr., M.Sc., Embrapa-Centro Nacional de Pesquisa de
}

Agrobiologia (CNPAB), Caixa Postal 74505, CEP 23890-000 Seropédica, RJ. E-mail: fdesouza@cnpab.embrapa.br

${ }^{3}$ Biól., Drạ, Instituto de Botânica, Caixa Postal 4005, CEP 01061-970 São Paulo, SP.

${ }^{4}$ Eng. Agr., Ph.D., Embrapa-CNPAB.

${ }^{5}$ Eng. Flor., Ph.D., Embrapa-CNPAB. 


\section{INTRODUÇÃO}

Os efeitos benéficos das associações micorrízicas sobre o crescimento de plantas, ciclagem de nutrientes e estruturação do solo estão amplamente demonstrados (Powell \& Bagyaraj, 1984; Varma \& Hock, 1995; Smith \& Read, 1997). Dentre os fungos formadores de micorrizas, os da ordem Glomales, a qual compreende exclusivamente os fungos micorrízicos arbusculares (FMAs), são especialmente importantes para as regiões tropicais. Em condições naturais, a grande maioria das espécies de plantas apresentam-se colonizadas por estes fungos, que potencializam a absorção de fósforo dos solos, e cujas características predominantes são os baixos teores de nutrientes disponíveis e alta capacidade de fixação de fosfatos (Mosse, 1981; Sieverding, 1989, 1991; Siqueira, 1994).

No entanto, os FMAs são biotróficos obrigatórios, e apresentam crescimento limitado quando cultivados axenicamente, sendo necessário o estabelecimento da colonização de raízes compatíveis e metabolicamente ativas para que o fungo possa se multiplicar (Azcón-Aguilar \& Barea, 1995; Bonfante \& Bianciotto, 1995). Sua propagação se dá através dos esporos, do micélio e de fragmentos de raízes colonizadas, coletivamente denominados propágulos, que ao infectarem as raízes da planta hospedeira, podem se desenvolver e dar origem à novos propágulos (Smith \& Read, 1997). Os propágulos que se apresentam ativos em um dado momento são denominados propágulos infectivos (PI). Em condições naturais, tem-se verificado que os fragmentos de raízes colonizadas e o micélio presente no solo são, em geral, mais infectivos do que os esporos. Estes aspectos foram revisados recentemente por Brundrett (1991) e Smith \& Read (1997). Já o termo potencial de inóculo é definido como o número de propágulos fúngicos viáveis, responsáveis pela infecção radicular inicial da planta hospedeira, ou seja, é um parâmetro que tem sido utilizado para avaliar a qualidade do inóculo (Liu \& Luo, 1994).

A principal forma de produção do inóculo ainda consiste no sistema tradicional de multiplicação do fungo em vasos-de-cultivo, que é um procedimento oneroso, demorado e de baixo rendimento. A inexistência de inoculantes comerciais no mercado brasileiro tem praticamente restringido a aplicação de FMAs a culturas que passem por uma fase em viveiro, dificultando a utilização destes microrganismos em larga escala.

Todavia, os FMAs são de ocorrência generalizada na maioria dos solos (Mosse, 1973; Trappe, 1987) e as práticas de manejo agrícolas provocam modificações qualitativas e quantitativas na população destes fungos simbiotróficos (Fairchild \& Miller, 1988; Dodd et al., 1990; Sieverding, 1991; Costa, 1993; Espíndola et al., 1998). Neste sentido, o conhecimento dos efeitos das práticas agrícolas sobre a população de FMAs indígenas consiste em uma das alternativas viáveis para aumentar os benefícios destes simbiontes para a produção agrícola. Segundo Abbott \& Robson (1991), o conhecimento da densidade de propágulos infectivos, capacidade infectiva e efetividade dos FMAs indígenas é fundamental para o desenvolvimento de estudos sobre ecologia e manejo destes fungos, bem como para auxiliar na introdução de fungos exóticos ao sistema.

Entre as metodologias empregadas para quantificação da ocorrência de FMAs, as mais utilizadas são: (a) extração e contagem de esporos (Gerdemann \& Nicolson, 1963); (b) avaliação da colonização radicular (Giovannetti \& Mosse, 1980); (c) estimativa da densidade de propágulos infectivos (esporos, micélio extra-radicular e fragmentos de raízes colonizadas) presentes no solo através da técnica do Número Mais Provável (NMP) (Porter, 1979). Estas metodologias apresentam pontos positivos e negativos e fornecem informações que muitas vezes se complementam (An et al., 1990; Abbott \& Robson, 1991).

A técnica de diluição denominada NMP foi idealizada por McCrady (1915) para estimar densidade da população microbiana sem necessidade de contagem direta. Ela é baseada na teoria das probabilidades e consiste na constatação da presença ou ausência de crescimento do microrganismo em porções individuais oriundas da diluição seriada de uma amostra de solo. De forma pioneira, Porter (1979) empregou a técnica do NMP para estimar o número de propágulos infectivos (NPI) de FMAs, e comparou os resultados com os obtidos pela técnica de extração de esporos por peneiramento via úmida e posterior contagem em microscópio estereoscópico. 
O autor concluiu que o NMP, por avaliar todos os propágulos que tem habilidade de colonizar a planta-teste, fornece uma avaliação mais precisa do potencial de inóculo quando comparado à técnica da extração de esporos, onde são avaliados, em geral, os esporos totais, não discriminando entre os viáveis (ativos e dormentes) e os inviáveis.

Vários autores já demonstraram que plantas afetam distintamente a população dos FMAs (Sieverding, 1991; Costa, 1993). Neste sentido, a utilização de pré-cultivos ou sistemas de rotação têm se mostrado alternativa viável para elevar o potencial de inóculo de populações de fungos indígenas (Ilag et al., 1987; Baltruschat \& Dehne, 1988, 1989; Harinikumar \& Bagyaraj, 1989; Dodd et al., 1990; Costa, 1993; Espíndola et al., 1998).

A adubação verde, notadamente com leguminosas, é uma prática em expansão, face ao grande potencial de produção de fitomassa, rusticidade e capacidade de fixar carbono e nitrogênio atmosférico, além de outras vantagens tais como o controle biológico de pragas e doenças, a melhoria das características físicas e químicas dos solos e a ciclagem de nutrientes (De-Polli et al., 1996). Seus efeitos sobre o potencial de inóculo de FMAs indígenas não têm sido devidamente investigados nos solos brasileiros (Espíndola et al.,1998).

Dentro deste contexto, a identificação de plantas com potencial para adubação verde deveria também considerar as mudanças relacionadas ao potencial de inóculo dos FMAs de forma a beneficiar a cultura subseqüente.

O objetivo deste trabalho foi avaliar a capacidade de pré-cultivos com leguminosas e uma gramínea em elevar a densidade de propágulos infectivos de FMAs indígenas do solo e seus efeitos sobre a produção da mandioca.

\section{MATERIAL E MÉTODOS}

O trabalho foi conduzido na Embrapa-Centro Nacional de Pesquisa de Agrobiologia (CNPAB), Seropédica (RJ), localizado a $22^{\circ} 46^{\prime}$ de latitude Sul e $43^{\circ} 41^{\prime}$ de longitude Oeste, com altitude de 33 metros, em um solo Podzólico Vermelho-Amarelo distrófico (PVd), textura franca, apresentando as seguintes características químicas na camada de $0-0,20 \mathrm{~m}$ de profundidade, de acordo com a metodologia descrita pela Embrapa (1979): $\mathrm{pH}$ em água $(1: 2,5) 5,6$; $\mathrm{Al}^{3+} 0,0 \mathrm{mmol}_{\mathrm{c}} \mathrm{dm}^{-3} ; \mathrm{Ca}^{2+} 27 \mathrm{mmol}_{\mathrm{c}} \mathrm{dm}^{-3} ; \mathrm{Mg}^{2+} 12 \mathrm{mmol}_{\mathrm{c}} \mathrm{dm}^{-3}$;
$\mathrm{K} 39 \mathrm{mg} \mathrm{dm}^{-3} ; \mathrm{P} 2,5 \mathrm{mg} \mathrm{dm}^{-3}$ de solo. A vegetação da área era composta predominantemente por capim-colonião (Panicum maximum Jacq.).

$\mathrm{O}$ experimento constou de dois ciclos de cultivo. No primeiro (pré-cultivo), foram semeadas as leguminosas feijão-de-porco (Canavalia ensiformis D.C.); mucuna-preta (Mucuna aterrima (Piper \& Tracy) Holland); crotalária (Crotalaria juncea L.); guandu (Cajanus cajan (L.) Millsp) e a gramínea sorgo (Sorghum bicolor (L.) Moench) cv. BR 005, além de um tratamento-controle (parcela mantida sem plantas). O segundo ciclo foi iniciado após o corte destas espécies, quando então se plantou mandioca (Manihot esculenta Crantz) cultivar Saracura.

O preparo inicial do solo constou de uma aração e três gradagens. Todas as espécies foram semeadas em sulcos distanciados por $0,50 \mathrm{~m}$. As densidades de plantio foram: 14 plantas para sorgo, 10 para crotalária e guandu e 6 para feijão-de-porco e mucuna-preta por metro. As parcelas correspondentes ao tratamento controle foram mantidas sem plantas através de capinas periódicas

As parcelas experimentais tinham $30 \mathrm{~m}^{2}$ e todas receberam adubação uniforme no sulco de plantio, equivalente a $26 \mathrm{~kg} \mathrm{ha}^{-1}$ de $\mathrm{P}$ na forma de termofosfato Yoorin e $33 \mathrm{~kg} \mathrm{ha}^{-1}$ de $\mathrm{K}$ na forma de $\mathrm{KCl}$. Todas as sementes de leguminosas foram infectadas com rizóbios específicos, fornecidos pela Embrapa-CNPAB. O plantio foi feito em dezembro de 1991 e o corte das plantas em março de 1992, correspondendo a 82 dias de crescimento. As plantas foram cortadas com rolo-faca acoplado a microtrator. A massa vegetal produzida foi deixada sobre a superfície do terreno como cobertura morta. Em seguida, manivas-sementes de mandioca da cultivar Saracura, com aproximadamente $0,20 \mathrm{~m}$ de comprimento, foram plantadas na posição horizontal em covas abertas sobre as mesmas linhas cultivadas anteriormente (pré-culturas), porém no espaçamento de $1 \mathrm{~m}$ entre linhas e $0,5 \mathrm{~m}$ entre plantas.

Amostras de solo foram coletadas em três épocas: a primeira, após o preparo inicial do solo (Dezembro, 1991); a segunda e a terceira, após o término, respectivamente, do 1 o ciclo de cultivo (Março, 1992) e do 2 o ciclo de cultivo (Novembro, 1992). Na primeira, uma amostra composta de 20 subamostras foi retirada da camada superficial $(0-0,20 \mathrm{~m})$ e nas seguintes, foram coletadas amostras compostas a partir da rizosfera de cinco plantas de cada parcela experimental. Também foi coletada uma amostra de raízes ao final de cada ciclo, para avaliação da taxa de colonização micorrízica. As raízes foram lavadas em água corrente e acondicionadas em frascos de plástico contendo solução alcoólica a $50 \%$, até serem processadas.

No primeiro ciclo, o peso da fitomassa aérea fresca foi determinado por amostragem, sendo retiradas duas amostras referentes a $1 \mathrm{~m}^{2}$ de área. Após pesagem, foi retirada 
uma subamostra para determinação do peso da amostra seca em estufa com circulação de ar forçado a $65^{\circ} \mathrm{C}$, até atingir massa constante. E no segundo ciclo, foram amostradas cinco plantas em cada parcela experimental.

Amostras de raízes foram clarificadas e coradas (Koske \& Gemma, 1989), para observação da presença de FMA e a taxa de colonização determinada pelo método da interseção de quadrantes, descrito em Giovanetti \& Mosse (1980). Os esporos foram extraídos de amostras com $50 \mathrm{~g}$ de solo seco ao ar, pela técnica de peneiramento em via úmida (Gerdemann \& Nicolson, 1963), identificados empregando-se um microscópio ótico equipado com câmara-clara e baseando-se fundamentalmente na morfologia, conforme descrito em Schenck \& Pérez (1988). Em outra amostra foram extraídos esporos e contados em placa canelada, com auxílio de microscópio estereoscópico, para determinação da freqüência de ocorrência de cada espécie. Os dados de porcentagem de colonização micorrízica e de freqüência foram transformados em arco sen $\sqrt{\mathrm{x} \cdot 100^{-1}}$ para a análise estatística.

A estimativa do número de propágulos infectivos (NPI) no início do experimento e após o primeiro ciclo de cultivo, foi determinada pela técnica do Número Mais Provável (NMP) em bioensaios montados sob condições de casa de vegetação, segundo a metodologia adaptada por Porter (1979), empregando-se o protocolo proposto por Sieverding (1991). Como planta teste, foi utilizada a leguminosa forrageira Stylosanthes guianensis, cultivar Schofield. Nas mesmas amostras foram avaliados o número de esporos, conforme metodologia descrita anteriormente. O NPI obtido pela técnica do NMP e o número de esporos obtidos pela técnica de peneiramento via úmida foram comparadas através de análise de correlação dos resultados obtidos após o primeiro ciclo de cultivo. $\mathrm{O}$ delineamento utilizado foi de blocos ao acaso com seis tratamentos e quatro repetições. Neste sentido, foram analisados 24 bioensaios para determinar o NMP, sendo um para cada parcela experimental, perfazendo um total de 960 amostras. As médias dos tratamentos foram comparadas através do teste de Duncan $(\mathrm{p}<0,05)$.

\section{RESULTADOS E DISCUSSÃO}

No primeiro ciclo de cultivo, não houve diferenças significativas na produção de matéria seca (MS) das espécies testadas (Tabela 1). Todas as leguminosas apresentaram colonização micorrízica, por ocasião do corte das plantas, significativamente superiores à obtida em sorgo (Tabela 2). A baixa taxa de colonização encontrada nas plantas de sorgo, possivelmente ocorreu pelo fato de estas já terem completado seu ciclo por ocasião da avaliação.

A taxa de colonização radicular na mandioca foi avaliada em plantas com oito meses de idade. Nesta fase, o efeito proporcionado pelos pré-cultivos sobre a colonização micorrízica das plantas de mandioca não resultou em diferenças significativas (Tabela 3). Em geral, diferenças no potencial de inóculo de solos ou inoculantes, sobre a taxa de colonização radicular, são detectados no início do ciclo de crescimento das plantas. Nesta fase, solos

TABELA 1. Produção de matéria seca da parte aérea de leguminosas e sorgo, após 82 dias de crescimento a campo. Média de quatro repetições.

\begin{tabular}{lc}
\hline Tratamento & $\begin{array}{c}\text { Matéria seca } \\
\left(\mathrm{t} \mathrm{ha}^{-1}\right)\end{array}$ \\
\hline Feijão-de-porco & $7,32 \mathrm{a}$ \\
Mucuna-preta & $5,42 \mathrm{a}$ \\
Crotalária & $4,94 \mathrm{a}$ \\
Guandu & $5,39 \mathrm{a}$ \\
Sorgo (palhada) & $4,41 \mathrm{a}$ \\
Sorgo (grãos + palhada) & $7,32 \mathrm{a}$ \\
\hline C.V. (\%) & 32,05 \\
\hline
\end{tabular}

1 Valores seguidos de letras diferentes diferem entre si pelo teste de Duncan $(\mathrm{p}<0,05)$.

TABELA2. Taxa de colonização radicular por fungos micorrízicos arbusculares em leguminosas e sorgo após 82 dias de crescimento. Média de quatro repetições ${ }^{1}$.

\begin{tabular}{lc}
\hline Tratamento & $\begin{array}{c}\text { Colonização } \\
(\%)\end{array}$ \\
\hline Feijão-de-porco & $52,8 \mathrm{a}$ \\
Mucuna-preta & $52,5 \mathrm{a}$ \\
Crotalária & $58,7 \mathrm{a}$ \\
Guandu & $51,4 \mathrm{a}$ \\
Sorgo & $39,1 \mathrm{~b}$ \\
\hline C.V. $(\%)$ & 10,0 \\
\hline
\end{tabular}

1 Valores seguidos de letras diferentes diferem entre si pelo teste de Duncan $(\mathrm{p}<0,05)$. 
que apresentem maior potencial de inóculo proporcionam uma colonização primária mais intensa (maior número de pontos de entrada). Posteriormente, com o desenvolvimento da colonização secundária estas diferenças tendem a se igualar (Walker \& Smith, 1984; Franson \& Bethlenfalvay, 1989). Este comportamento foi relatado por Siqueira et al. (1994) para mudas de cafeeiro inoculadas com quantidades crescentes de esporos. Os autores reportaram que diferenças marcantes na taxa de colonização só foram detectadas até 30 dias após a inoculação. Aos 60 dias, a colonização em todos os tratamentos, se apresentava em torno do máximo e aos 140 dias, a taxa de colonização não diferiu quanto à quantidade de esporos empregada. Estes autores concluíram que a capacidade máxima do cafeeiro em formar micorrizas, nas condições do estudo, foi atingida com 50 esporos por planta.

A produtividade de raízes tuberosas de mandioca não foi afetada significativamente pelos pré-cultivos (Tabela 3). A produção de folhas foi superior nas parcelas pré-cultivadas com as leguminosas mucuna e crotalária, em comparação com o sorgo e o controle. O mesmo ocorreu quanto à produção de ramas em relação às parcelas pré-cultivadas com sorgo. O desenvolvimento da parte aérea da mandioca é favorecido pelo aumento na oferta de $\mathrm{N}$ no solo, o que pode reduzir a produção de raízes tuberosas, por prejudicar o direcionamento de re- servas para os órgãos de armazenamento (Howeler, 1982). Neste caso, o aporte de N via fixação biológica de $\mathrm{N}$ pelas leguminosas mucuna e crotalária pode ter favorecido o desenvolvimento da parte aérea das plantas de mandioca, enquanto o sorgo retirou parte do $\mathrm{N}$ do solo para a produção de grãos, promovendo um efeito contrário (Souza, 1996). Em função disto, a rotação de culturas com estas leguminosas, poderá ser recomendada para solos pobres em $\mathrm{N}$, e o uso do sorgo para solos com maiores teores deste elemento.

Foram identificadas 16 espécies de FMA na área experimental, e constatou-se que algumas espécies sofreram significativa alteração na freqüência de distribuição, em função dos dois ciclos de cultivo (Tabela 4). Entre as espécies encontradas, o Glomus etunicatum apresentou a maior freqüência de ocorrência, nos dois ciclos avaliados. No entanto, após o cultivo da mandioca sua ocorrência decresce em $37,4 \%$. Com exceção do Glomus invermaium e Scutellospora weresubiae, as demais espécies de Glomus, Gigaspora e Scutellospora apresentaram tendência de redução após o cultivo da mandioca; fato contrário ocorreu com as espécies de Acaulospora, que aumentaram, com exceção da A. foveata; tais resultados estão de acordo com os relatados por Sieverding (1991).

A maioria dos FMAs não apresenta especificidade hospedeira (Morton, 1988; Sieverding,

TABELA 3. Taxa de colonização radicular por fungos micorrízicos arbusculares, produtividade e produção de folhas, ramos e tubérculos de plantas de mandioca, colhidas com oito meses, em áreas previamente cultivadas com leguminosas e sorgo. Médias de quatro repetições ${ }^{1}$.

\begin{tabular}{|c|c|c|c|c|c|}
\hline \multirow{2}{*}{ Tratamento } & \multirow{2}{*}{$\begin{array}{l}\text { Colonização } \\
\text { radicular (\%) }\end{array}$} & \multirow{2}{*}{$\begin{array}{l}\text { Produtividade } \\
\left(\mathrm{t} \mathrm{ha}^{-1}\right)\end{array}$} & Folhas & Ramas & Tubérculos \\
\hline & & & \multicolumn{3}{|c|}{ Peso de matéria seca $\left(\mathrm{kg} \mathrm{planta}^{-1}\right)$} \\
\hline Feijão-de-porco & $62,60 \mathrm{a}$ & $12,76 \mathrm{a}$ & $0,68 \mathrm{ab}$ & $1,17 \mathrm{ab}$ & $0,64 \mathrm{a}$ \\
\hline Mucuna-preta & $57,80 \mathrm{a}$ & $15,20 \mathrm{a}$ & $0,74 \mathrm{a}$ & $1,31 \mathrm{a}$ & $0,76 \mathrm{a}$ \\
\hline Crotalária & $54,42 \mathrm{a}$ & $16,72 \mathrm{a}$ & $0,73 \mathrm{a}$ & $1,32 \mathrm{a}$ & $0,84 \mathrm{a}$ \\
\hline Guandu & $61,12 \mathrm{a}$ & $14,32 \mathrm{a}$ & $0,63 a b$ & $1,12 \mathrm{ab}$ & $0,72 \mathrm{a}$ \\
\hline Sorgo & $46,51 \mathrm{a}$ & $17,20 \mathrm{a}$ & $0,50 \mathrm{~b}$ & $0,82 \mathrm{~b}$ & $0,86 \mathrm{a}$ \\
\hline Sem vegetação & $58,30 \mathrm{a}$ & $12,96 \mathrm{a}$ & $0,51 \mathrm{~b}$ & $1,01 \mathrm{ab}$ & $0,65 \mathrm{a}$ \\
\hline C.V. $(\%)$ & 19,94 & 17,95 & 18,19 & 19,08 & 17,95 \\
\hline
\end{tabular}

1 Valores seguidos de letras diferentes na mesma coluna diferem entre si pelo teste de Duncan $(\mathrm{p}<0,05)$. 
1989). Contudo, normalmente ocorre uma seletividade (habilidade discriminatória) entre as espécies de uma determinada população de FMAs e o macrossimbionte (Sieverding, 1989), por influência das condições edafoclimáticas (Saif, 1986), provocando variações com o cultivo nas espécies de uma determinada população. Trabalhos conduzidos na Colômbia demonstraram que anos sucessivos de monocultura de mandioca favoreceram a multiplicação de Acaulospora spp., e que o aumento destas espécies foi associado à redução da produtividade da cultura (Sieverding, 1989). Práticas de cultivo, adubação e a calagem também são fatores determinantes desta seletividade (Schenck \& Kinlock, 1980; Daniel Hentrick \& Bloon, 1983).

O número inicial de esporos (determinados por extração e contagem) e de propágulos infectivos (determinados pela metodologia do NMP) após o preparo do solo foram, respectivamente, 479 esporos $100 \mathrm{~g}^{-1}$ e 129 (61 - 277) propágulos $100 \mathrm{~g}^{-1}$ de solo seco.
Quando se contrastam estes valores com os obtidos após os pré-cultivos, verifica-se a tendência de decréscimo no tratamento controle em relação à amostragem inicial (Fig. 1). Os demais tratamentos, com exceção da crotalária, proporcionaram tendências de aumento do NPI, e houve um destaque quanto às parcelas pré-cultivadas com sorgo, o que demonstra que, apesar de o ciclo de crescimento das plantas ter sido relativamente curto (82 dias), esta prática pode resultar no incremento do NPI.

Verifica-se que, no início do experimento, o número de esporos estava relativamente alto e o NPI apresentava-se baixo, quando comparado com os valores obtidos no final do ciclo. Esta aparente discrepância é comum, e tem sido atribuída à presença de grande número de esporos inativos e dormentes (Porter, 1979; An et al., 1990), podendo também indicar um efeito depressivo, causado pelo revolvimento do solo através da aração e gradagem, sobre a capacidade infectiva da malha de hifas da população de

TABELA 4. Freqüência de ocorrência das espécies de fungos micorrízicos arbusculares, avaliada a partir de esporos coletados na ocasião do preparo inicial do solo e após dois ciclos de cultivo, o primeiro com 82 dias de crescimento de leguminosas e sorgo, e o segundo, com oito meses de cultivo de mandioca cultivar Saracura. Médias de 4 repetições¹.

\begin{tabular}{lrrr}
\hline Espécies & \multicolumn{3}{c}{ Freqüência (\%) } \\
\cline { 2 - 4 } & Inicial & $1^{\circ}$ ciclo & $2^{\mathrm{o}}$ ciclo \\
\hline Acaulospora foveata Trape \& Gerd. & 0,2 & $0,7 \mathrm{a}$ & $0,4 \mathrm{a}$ \\
A. longula Spain \& Schenck & - & $0,3 \mathrm{a}$ & $0,6 \mathrm{a}$ \\
A. mellea Spain \& Schenck & 4,0 & $0,0 \mathrm{~b}$ & $8,0 \mathrm{a}$ \\
A. morrowae Spain \& Schenck & 2,3 & $1,5 \mathrm{a}$ & $4,8 \mathrm{a}$ \\
A. scrobiculata Trappe & 5,6 & $1,0 \mathrm{~b}$ & $16,4 \mathrm{a}$ \\
A. tuberculata Janos \& Trappe & 0,2 & $0,1 \mathrm{a}$ & $0,4 \mathrm{a}$ \\
Gigaspora ramisporophora Spain, Sieverding \& Schenck & 2,6 & $1,4 \mathrm{a}$ & $0,3 \mathrm{a}$ \\
Glomus etunicatum Becker \& Gerd. & 37,3 & $54,0 \mathrm{a}$ & $33,8 \mathrm{~b}$ \\
G. geosponim (Nicol. \& Gerd.) Walker & 0,4 & $0,3 \mathrm{a}$ & $0,0 \mathrm{a}$ \\
G. invermaium Hall & 4,2 & $2,8 \mathrm{~b}$ & $8,6 \mathrm{a}$ \\
G. macrocarpum Tul. \& Tul. & 17,6 & $10,9 \mathrm{a}$ & $6,6 \mathrm{a}$ \\
G. sinuosum (Gerd. \& Bakshi) Almeida \& Schenck & 5,6 & $0,0 \mathrm{a}$ & $0,0 \mathrm{a}$ \\
G. sp1 & 1,8 & $0,3 \mathrm{a}$ & $0,0 \mathrm{a}$ \\
G. sp2 & - & $19,1 \mathrm{a}$ & $11,1 \mathrm{~b}$ \\
Scutellospora heterogama (Nicol. \& Gerd.)Walker \& Sanders & 10,0 & $4,7 \mathrm{a}$ & $2,5 \mathrm{a}$ \\
S. weresubiae Koske \& Walker & 4,6 & $1,4 \mathrm{~b}$ & $6,0 \mathrm{a}$ \\
Não identificados & 3,6 & $0,6 \mathrm{a}$ & $0,7 \mathrm{a}$ \\
\hline
\end{tabular}

1 Valores seguidos de letras diferentes na mesma linha diferem entre si pelo teste de Duncan $(p<0,05)$ 
FMAs indígenas (Moorman \& Reeves, 1979; Reeves et al., 1979; Evans \& Miller, 1988, 1990; Jasper et al., 1989). Nesta condição, a avaliação sobre NPI parece ser mais sensível às alterações impostas pelo manejo empregado (efeitos deletérios provocados pelo preparo do solo) do que a avaliação sobre o número de esporos.

Os valores encontrados com relação ao número de propágulos infectivos no início e ao final do experimento estão na mesma faixa dos encontrados em outros trabalhos (Ilag et al., 1987; Sieverding, 1991). Contudo, Harinikumar \& Bagyaraj $(1988,1989)$ relataram, ao se referirem a solos da Índia, valores bem maiores, o que pode ser atribuído a vários fatores como: o tipo, umidade e manejo do solo, modelo de produção, capacidade de suporte das plantas hospedeiras utilizadas, presença de predadores naturais dos FMAs, capacidade infectiva e número inicial de propágulos da população indígena dos FMAs, além da duração do ciclo de crescimento das plantas (Ilag et al., 1987; Simpson \& Daft, 1990).

Entre as espécies testadas, o sorgo promoveu um aumento significativo no NPI em relação ao tratamento-controle. Entre as leguminosas, embora o feijão-de-porco, a mucuna-preta e o guandu tenham apresentado valores com tendência de aumento no NPI em relação ao tratamento-controle, estes não alcançaram diferenças significativas (Fig. 1). Em sorgo, a colonização micorrízica e a esporulação foram menores do que nas leguminosas, e o NPI foi alto (Fig. 1), o que evidencia que, na época da avaliação, a principal forma de inóculo consistia de micélio fúngico presente no solo e nas raízes colonizadas (Smith \& Read, 1997). Apesar de as raízes do sorgo apresentarem menor taxa de colonização, o sistema radicular desta planta é, em geral, maior e mais ramificado, e apresenta maior quantidade de radículas finas do que o das leguminosas testadas. Assim, o elevado NPI no sorgo deve estar relacionado ao comprimento do sistema radicular desta planta. Por outro lado, no tocante aos tratamentos ausência de vegetação e leguminosas, o NPI apresentou correlação significativa com o número de esporos (Fig. 2), evidenciando que nestes tratamentos a forma predominante de propágulos de FMAs consistia de esporos.

Geralmente, espécies de gramíneas, como milho, sorgo, milheto e Brachiaria decumbens, favorecem mais o aumento da esporulação e do NPI do que espécies de leguminosas (Ilag et al.,1987; Simpson \& Daft, 1990; Reis et al., 1991), e esta diferença tem sido atribuída ao maior comprimento de raízes

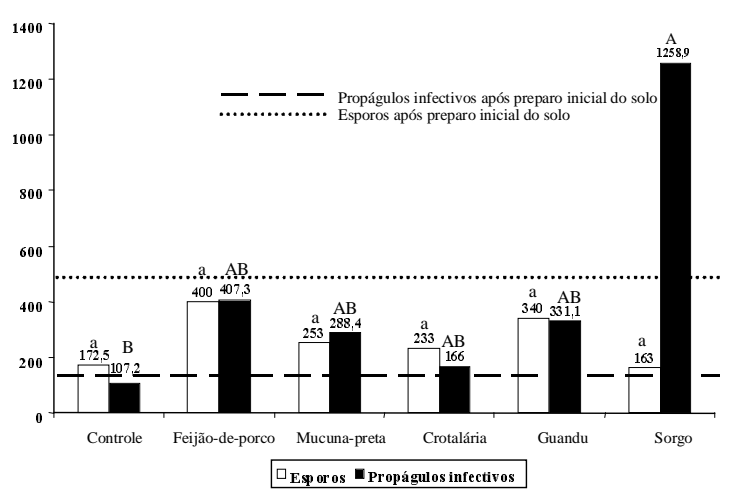

FIG. 1. Número de esporos (……..) e de propágulos infectivos (------) de fungos micorrízicos arbusculares no início (preparo do solo) e após 82 dias de cultivo com feijão-de-porco (F), mucuna (M), crotalária $(C)$, guandu $(G)$ e sorgo (S) em relação ao tratamento controle (ausência de vegetação). Letras minúsculas e maiúsculas representam a comparação entre médias, respectivamente, para esporos e propágulos infectivos pelo teste de Duncan $(\mathbf{P}>\mathbf{0 , 0 5})$.

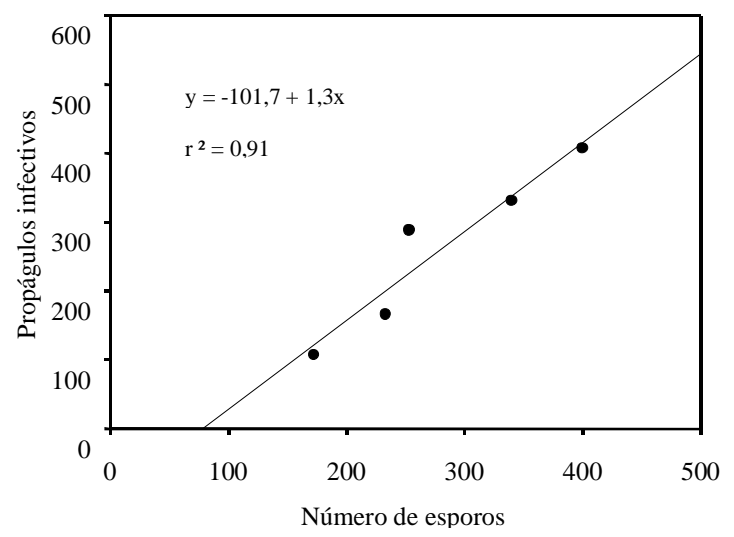

FIG. 2. Correlação entre número de esporos e de propágulos infectivos de fungos micorrízicos arbusculares (por $100 \mathrm{~g}$ de solo seco) após 82 dias de cultivo de leguminosas (crotalária, guandu, feijão-de-porco e mucuna-preta) e nas parcelas-controle (ausência de vegetação). 
infectadas das gramíneas. Por outro lado, Harinikumar \& Bagyaraj (1988) relacionaram o melhor desempenho de plantas de amendoim em relação ao milheto e ao girassol às características do sistema radicular desta planta, ligadas à dependência micorrízica, por estes últimos serem grosseiros e com poucos pêlos absorventes. No tratamento-controle ocorreu o oposto do cultivado com sorgo. Na ausência de vegetação, a principal forma de propágulos foram os esporos, que aparentemente mantiveram a viabilidade durante o período de pousio.

Outra possível explicação para o elevado potencial de inóculo encontrado após o cultivo do sorgo seria devido à planta teste utilizada no bioensaio. Quando esta é originária do mesmo sítio de coleta do solo em estudo, ela tende a fornecer valores mais altos (Adelman \& Morton, 1986), porém neste estudo foi empregado como planta teste nos bioensaios do NMP o estilosantes, por não ter sido utilizado nos pré-cultivos e por apresentar alta dependência micorrízica e sementes pequenas (Sieverding, 1991).

Após o cultivo das leguminosas e nas parcelascontrole, verificou-se correlação entre o NE e o NPI, onde estas duas técnicas forneceram valores próximos. Quando os dados referentes às parcelas cultivadas com sorgo são incluídos, os valores não se correlacionam, e isto demonstra que para esta gramínea, na condição avaliada, o número de esporos não reflete o NPI. Fica, assim, constatado que a técnica do NMP detectou mudanças provocadas pelas práticas agrícolas sobre o NPI de FMAs, em situações onde a técnica de contagem de esporos não foi sensível. Embora a técnica do NMP apresente melhor avaliação do NPI do solo, ela ainda é limitada, uma vez que no preparo do bioensaio é necessário manuseio intenso do solo para homogeneização das amostras, o que acarreta destruição de parte do micélio fúngico, causando assim redução da capacidade infectiva do solo (Evans \& Miller, 1988, 1990; Jasper et al., 1989).

A otimização da população de FMAs indígenas é uma alternativa à falta de inoculantes em quantidade e qualidades adequadas. A viabilidade desta técnica foi mostrada neste trabalho, com a identificação de plantas com potencial para alterar o NPI. Porém, estudos mais detalhados sobre a ecologia e manejo destes simbiontes são necessários a fim de se otimizar a população de FMAs indígenas não só quantitativamente mas também de forma qualitativa.

A falta de metodologias adequadas a este tipo de estudo e a necessidade de técnicos habilitados a identificar taxonomicamente estes fungos são alguns dos pontos que dificultam avanços da pesquisa nesta área. Neste sentido, o desenvolvimento e a validação de metodologias apropriadas ao estudo destes simbiontes a campo é fundamental. A técnica do NMP utilizada neste estudo, apesar de suas limitações, apresentou sensibilidade para evidenciar os efeitos de práticas agrícolas sobre a população de FMAs indígenas.

\section{CONCLUSÕES}

1. O pré-cultivo com as leguminosas e com o sorgo eleva a produção de folhas e de ramos, respectivamente, mas não afeta a produção de raízes de mandioca.

2. O pré-cultivo com sorgo eleva o número de propágulos infectivos.

3. Não há relação entre aumento no número de propágulos e a produção ou micorrização das plantas de mandioca colhidas com oito meses de cultivo.

4. A forma de propágulo de fungos micorrízicos arbusculares predominante no solo após o primeiro ciclo de cultivo são esporos, exceto nas parcelas précultivadas com sorgo.

5. A freqüência de ocorrência das espécies de fungos micorrízicos arbusculares sofre variação com os ciclos de cultivo.

\section{AGRADECIMENTOS}

À Embrapa e à FAPERJ, pelo apoio financeiro (PNP - Embrapa cod. 04092011/8), e à CAPES, pela concessão de bolsa de estudo ao primeiro autor.

\section{REFERÊNCIAS}

ABBOTT, L.K.; ROBSON, A.D. Factors influencing the occurrence of vesicular-arbuscular mycorrhizas Agriculture, Ecosystems and Environment, Amsterdan, v.35, p.121-150, 1991 
ADELMAN, M.J.; MORTON, J.B. Infectivity of vesicular-arbuscular mycorrhizal fungi: influence of host-soil diluent combination on MPN estimates and percentage colonization. Soil Biological \& Boichemistry, Great Britain, v.18, p.77-83, 1986.

AN, Z.Q.; HENDRIX, J.W.; HERSHMAN, D.E.; HENSON, G.T. Evaluation of the "most probable number" (MPN) and wet-sieving methods for determining soil-borne population of endogonaceous mycorrhizal fungi. Mycologia, New York, v.82, n.5 p.576-581, 1990.

AZCÓN-AGUILAR, C.; BAREA, J.M. Saprophytic growth of arbuscular mycorrhizal fungi. In: VARMA, A.; HOCK, B. (Eds.). Mycorrhiza, structure, function, molecular biology and biotechnology . Berlin: Springer-Verlag, 1995. p.391407.

BALTRUSCHAT, H.; DEHNE, H.W. The occurrence of vesicular-arbuscular mycorrhizal in agroecosystems. I. Influence of nitrogen fertilization and green manure in continuous monoculture and in crop rotation on the inoculum potential of winter wheat Plant and Soil, Dordrecht, v.107, p.279-284, 1988

BALTRUSCHAT, H.; DEHNE, H.W. The occurrence of vesicular-arbuscular mycorrhizal in agroecosystems. II. Influence of nitrogen fertilization and green manure in continuous monoculture and in crop rotation on the inoculum potential of winter barley. Plant and Soil, Dordrecht, v.113, p.251-256, 1989.

BONFANTE, P.; BIANCIOTTO, V. Presymbiotic versus symbiotic phase in arbuscular endomycorrhizal fungi: morphology and cytology. In: VARMA, A.; HOCK, B. (Eds.). Mycorrhiza: structure, function, molecular biology and biotechnology. Berlin: Springer-Verlag, 1995. p.229-247

BRUNDRETT, M. Mycorrhizas in natural ecosystems. Advances in Ecological Research, Toronto, v.21, p.171-313, 1991.

COSTA, S.M.G. Fungos micorrízicos arbusculares em monocultura e rotações de milho (Zea mays L.) e soja (Glycine max (L.) Merrill). Rio Claro, SP UNESP, 1993. 129p. Tese de Doutorado.

DANIEL HENTRICK, B.A.; BLOON, J. Vesicular arbuscular mycorrhizal fungi associated with native tall grass prairie and cultivated with wheat. Canadian Journal of Botany, Ottawa, v.61, p.2140-2146, 1983
DE-POLLI, H.; GUERRA, J.G.M.; ALMEIDA, D.L. de; FRANCO, A.A. Adubação verde: parâmetros para avaliação de sua eficiência. In: CONGRESSO BRASILEIRO E ENCONTRO NACIONAL DE PESQUISA SOBRE CONSERVAÇÃO DO SOLO, 8., 1990, Londrina. Manejo integrado de solos e microbacias hidrográficas. Londrina: IAPAR, 1996. p.225-243.

DODD, J.C.; ARIAS, I.; KOOMEN, I.; HAYMAN, D.S. The management of populations of vesiculararbuscular mycorrhizal fungi in acid-infertile soils of a savanna ecosystem. Plant and Soil, Dordrecht, v.122, p.241-247, 1990

EMBRAPA. Serviço Nacional de Levantamento e Conservação de Solos (Rio de Janeiro, R.J). Manual de métodos de análise de solo. Rio de Janeiro, 1979. Não paginado.

ESPÍNDOLA, J.A.A.; ALMEIDA, D.L. de; GUERRA, J.G.M.; SILVA, E.M.R. da; SOUZA, F.A. de. Influência da adubação verde na colonização micorrízica e na produção da batata-doce. Pesquisa Agropecuária Brasileira, Brasília, v.33, n.3 p.339-347, 1998.

EVANS, D.G.; MILLER, M.H. The role of the external mycelial network in the effect of soil disturbance upon vesicular-arbuscular mycorrhizal colonization of maize. New Phytologist, Oxford, v.114, p.65-71, 1990

EVANS, D.G.; MILLER, M.H. Vesicular-arbuscular mycorrhizas and the soil-disturbance-induced reduction of nutrient absorption in maize. I. Casual relations. New Phytologist, Oxford, v.110, p.6774,1988

FAIRCHILD, G.L; MILLER, M.H. Vesicular-arbuscular mycorrhizas and the soil-disturbance-induced reduction of nutrient absortion in maize II. Development of the effect. New Phytologist, Oxford, v.110, p.75-84, 1988

FRANSON, R.L.; BETHLENFALVAY, G.J. Infection unit method of vesicular-arbuscular mycorrhizal propagule determination. Soil Science Society of American. Journal, v.53, p.754-756, 1989

GERDEMANN, J.W.; NICOLSON, T.H. Espores of mycorrhizal Endogone species extracted from soil by wet-sieving and decanting. Transactions of the British Mycological Society, Cambridge, v.46, p.235-244, 1963. 
GIOVANNETTI, M.; MOSSE, B. An evaluation of techniques for measuring vesicular arbuscular mycorrhizal infection in roots. New Phytologist, Oxford, v.84, p.489-500, 1980

HARINIKUMAR, K.M.; BAGYARAJ, D.J. Effect of crop rotation on native vesicular arbuscular mycorrhizal propagules in soil. Plant and Soil, Dordrecht, v.110, p.77-80, 1988

HARINIKUMAR, K.M.; BAGYARAJ, D.J. Effect of crop sequence, fertilizers and farmyard manure on vesicular-arbuscular mycorrhizal fungi in different crop over three consecutive seasons. Biology and Fertility of Soils, Berlin, v.7, p.173-175, 1989.

HOWELER, R.M. Mineral nutrition and fertilization of cassava. In: COCK, J.H.; REYES, J.A. (Eds.). Cassava: research, production and utilization. Cali: UNDP/CIAT, 1982. p.745-761.

ILAG, L.L.; ROSALES, A.M.; ELAZEGUI, F.A.; MEW, T.W. Changes in the population of infective endomycorrhizal fungi in a rice-based cropping system. Plant and Soil, Dordrecht, v.103, p.67-73, 1987.

JASPER, D.A.; ABBOTT, L.K.; ROBSON, A.D. Soil disturbance reduces the infectivity of external hyphae of vesicular-arbuscular mycorrhizal fungi. New Phytologist, Oxford, v.112, p.93-99, 1989.

KOSKE, R.E.; GEMMA, J.N. A modified procedure for staining roots to detect VA mycorrhizas. Mycological Research, Cambridge, v.92, p.488-505, 1989.

LIU, R.-J.; LUO, X.-S. A new method to quantify the inoculum potential of arbuscular mycorrhizal fungi. New Phytologist, Oxford, v.128, p.89-92, 1994.

McCRADY, M.H. The numerical interpretation of fermantation-tubes results. Journal of Infection Disease, Illinois, v.17, p.183-212, 1915

MOORMAN, T.; REEVES, F.B. The role of endomycorrhizae in revegetation practices in the semi-arid west. II. A bioassay to determine the effect of land disturbance on endomycorrhizal populations. American Journal of Botany, v.66, n.1, p.14-18, 1979

MORTON, J.B. Taxonomy of VA mycorrhizal fungi: Classification, nomenclature and identification. Mycotaxon, Ithaca, v.32, p.267-324, 1988.
MOSSE, B. Advances in the study of vesicular-arbuscular mycorrhizal. Annual Review of Phytopathology, Palo Alto, v.11, p.171-196, 1973.

MOSSE, B. Vesicular-arbuscular mycorrhizal research for Tropical Agriculture. Hawaii: Hawaii Institute of Tropical Agricultural and Human Resources, University of Hawaii, 1981. 82p (Research Bulletin, 194).

PORTER, W.M. The "Most Probable Number" method for enumerating infective propagules of vesicular arbuscular mycorrhizal fungi in soil. Australian Journal of Soil Research, Victoria, v.17, p.515-519, 1979.

POWELL, C.L.; BAGYARAJ, D.J. VA mycorrhiza. Boca Raton: CRC, 1984. 234p

REEVES, F.B.; WAGNER, D.; MOORMAN, T.; KIEL, $\mathrm{J}$. The role of endomycorrhizae in revegetation pratices in the semi-arid. west. I. A comparison of incidence of mycorrhizae in severely disturbed vs. natural environments. American Journal of Botany, v.66, p.6-13, 1979

REIS, M.A.; ZAMBOLIM, L.; NASCIMENTO, J.R do. Potencial de utilização de gramíneas para multiplicação de inóculo de fungos micorrízicos vesículo arbucular Glomus etunicatum. Fitopatologia Brasileira, Brasília, v.16, n.3, p.164-170, 1991.

SAIF, S.R. Vesicular-arbuscular mycorrhizae in tropical forage species as influenced by season, soil texture, fertilizers, host species and ecotypes. Angewandte Botanik, Berlin, v.60, p.125-139, 1986

SCHENCK, N.C.; KINLOCK, R.A. Incidence of mycorrhizal fungi on six field crops in monoculture on newly cleared woodland site. Mycologia, New York, v.72, p.445-456, 1980.

SCHENCK, N.C.; PÉREZ, Y. Manual for the identification of VA mycorrhizal fungi. 2.ed. Gainesville: INVAM, Plant Pathology Department and Agriculture Sciences, Univ. of Florida, 1988 $241 \mathrm{p}$

SIEVERDING, E. Ecology of VAM fungi in tropical agrosystems. Agriculture, Ecosystems and Environment, Amsterdan, v.29, p.369-390, 1989.

SIEVERDING, E. Vesicular-arbuscular mycorrhiza management in tropical agrosystems. Eschborn, Fed. Rep. of Germany: Friedland Bremer, 1991. $371 \mathrm{p}$. 
SIMPSON, D.; DAFT, M.J. Spore production and mycorrhizal development in various tropical crop host infected with Glomus clarum. Plant and Soil, Netherlands, v.121, p.117-178, 1990

SMITH, S.E.; READ, D.J. Mycorrhizal symbiosis. 2.ed. San Diego, California: Academic, 1997.605p.

SIQUEIRA, J.O. Micorrizas arbusculares. In: ARAÚJO, R.S.; HUNGRIA, M. (Eds.). Microorganismos de importância agrícola. Brasília: Embrapa-CNPAF/ Embrapa-CNPSo, 1994. p.151-194.

SIQUEIRA, J.O.; COLOZZI FILHO, A.; SAGGIN JUNIOR, O. Efeito da infecção de plântulas de cafeeiro com quantidades crescentes de esporos do fungo endomicorrízico Gigaspora margarita. Pesquisa Agropecuária Brasileira, Brasília, v.29, n. 6, p. $875-883,1994$

SOUZA, F.A. de. Influência da adubação verde sobre o potencial de inóculo de fungos micorrízicos arbusculares e produção da mandioca (Manihot esculenta, Crantz). Seropédica: UFRRJ, 1996. 88p Tese de Mestrado.

TRAPPE, J.M. Phylogenetic and ecologic aspects of mycotrophy in the Angiosperms from an evolutionary standpoint. In: SAFIR, G.R. (Ed.). Ecophysiology of VA mycorrhizal plants. Boca Raton: CRC, 1987. p.5-25.

VARMA, A.; HOCK, B. (Eds.). Mycorrhiza: structure, function, molecular biology and biotechnology. Berlin: Springer-Verlag, 1995. 747p.

WALKER, N.A.; SMITH, S.E. The quantitative study of mycorrhizal infection. II. The relation of rate of infection and speed of fungal growth to propagule density, the mean lenght of infection unit and the limiting value of the fraction of root infected. New Phytologist, Oxford, v.96, p.55-69, 1984. 